\title{
Understanding colloidal charge renormalization from surface chemistry: Experiment and theory
}

\author{
T. Gisler, S. F. Schulz, M. Borkovec, and H. Sticher \\ Institute of Terrestrial Ecology, Federal Institute of Technology (ETH), Grabenstrasse 3, \\ CH-8952 Schlieren, Switzerland \\ P. Schurtenberger \\ Polymer Institute, Federal Institute of Technology (ETH), CH-8092 Zürich, Switzerland \\ B. D'Aguanno \\ Center for Advanced Studies in Sardinia (CRS4), 91000 Cagliari, Italy \\ R. Klein \\ Faculty of Physics, University of Konstanz, D-78434 Konstanz, Germany \\ (Received 6 May 1994; accepted 10 August 1994)
}

\begin{abstract}
In this paper we report on the charging behavior of latex particles in aqueous suspensions. We use static light scattering and acid-base titrations as complementary techniques to observe both effective and bare particle charges. Acid-base titrations at various ionic strengths provide the $p \mathrm{H}$ dependent charging curves. The surface chemical parameters (dissociation constant of the acidic carboxylic groups, total density of ionizable sites and Stern capacitance) are determined from fits of a Stern layer model to the titration data. We find strong evidence that the dissociation of protons is the only specific adsorption process. Effective particle charges are determined by fits of integral equation calculations of the polydisperse static structure factor to the static light scattering data. A generalization of the Poisson-Boltzmann cell model including the dissociation of the acidic surface groups and the autodissociation of water is used to predict effective particle charges from the surface chemical parameters determined by the titration experiments. We find that the light scattering data are best described by a model where a small fraction of the ionizable surface sites are sulfate groups which are completely dissociated at moderate $p \mathrm{H}$. These effective charges are comparable to the predictions by a basic cell model where charge regulation is absent.
\end{abstract}

\section{INTRODUCTION}

The study of structure and dynamics of aqueous colloidal suspensions has received considerable attention in the past few years. Mainly scattering experiments and theoretical studies based on concepts from liquid state theory have contributed much to the understanding of the relationship between the microscopic structure and the macroscopic properties of these systems. ${ }^{1}$ The aqueous surface chemistry of such colloidal particles, on the other hand, has been the subject of many investigations, where methods of classical electrochemistry and concepts of electrical double layer theory ${ }^{2}$ have essentially helped to understand processes such as ion exchange and surface complexation. ${ }^{3}$

However, the relationship between the structural approach from liquid state theory and the chemical equilibrium approach stressed in surface chemistry has not been fully understood, also because of the lack of complementary experimental data on well-characterized model systems. Especially the physical interpretation of the effective particle charge observed in scattering experiments is still a controversial issue. In the analysis of scattering data the effective charge is usually treated as an adjustable parameter in the Yukawa potential used to model the screened Coulomb interaction between the macroions. The bare charge resulting from the dissociation of protons from acidic surface groups, on the other hand, can be directly observed in acid-base titrations.
The analysis of small angle neutron scattering data of micellar systems (see the work by Chen and Sheu ${ }^{4}$ for a recent review) has provided evidence that the effective charge appearing in the Yukawa potential has, in general, to be related to the bare surface charge by some renormalization procedure. This idea has been amply discussed in the literature on polyelectrolytes ${ }^{5}$ as the so-called Manning condensation or counterion condensation. Bucci et al. ${ }^{6}$ have recently used ionic-nonionic mixed micelles to vary systematically the surface charge density at constant particle size. Their effective charges show nicely the crossover to a saluration value as the bare charge increases. Versmold et al. ${ }^{7}$ were among the first to relate the effective charge to the chemistry at the particle surface. They measured static structure factors of suspensions to which $\mathrm{NaOH}$ and $\mathrm{NaCl}$, respectively, had been added. The structure was found to remain unchanged by the addition of $\mathrm{NaOH}$, which was attributed to an exchange of bound protons by sodium ions; the released protons being neutralized by the added hydroxide. Addition of $\mathrm{NaCl}$, on the other hand, resulted in a pronounced loss of structure due to the elevated ionic strength. The marked underestimation of the bare charge by the effective charge measured in the static light scattering experiments was attributed to condensation of counterions onto the charged particle surface.

In a different way the present work addresses the relationship between bare and effective charge of colloidal par- 
ticles. We use polystyrene latex particles carrying ionizable carboxylic surface groups as a model system. We determine the surface chemical parameters (equilibrium constant of the surface dissociation reaction and the number of ionizable sites) from acid-base titrations. These quantities are used in a generalized Poisson-Boltzmann cell model including surface dissociation and the autodissociation of water to calculate the distribution of the mean electrostatic potential around a particle. By the charge renormalization procedure of Alexander et al. ${ }^{8}$ we predict the effective charges. These, in turn, can be compared with the effective charges resulting from the analysis of static light scattering data, properly taking into account the polydispersity in size and charge.

The complementary use of titration and light scattering experiments is reflected in the organization of the paper: In Sec. II we briefly review integral equation methods from the statistical mechanics of charged colloidal suspensions. We then recall concepts from surface chemistry to describe the interface between the solid and the electrolyte solution in the presence of acidic surface groups. Section II C is concerned with the Poisson-Boltzmann cell model including surface dissociation. In Sec. III we present the details of the particle characterization, the structure factor determination with static light scattering, and the titration experiments. Finally, we compare the experimental data with the predictions of the generalized Poisson-Boltzmann cell model.

\section{THEORY}

\section{A. Colloidal equilibrium structure}

In this section we briefly summarize the integral equation theory approach for the description of the equilibrium structure of a colloidal suspension. Since there is a close analogy between a colloidal suspension and a classical simple liquid, ${ }^{9}$ technical details may be found in standard texts on liquid state theory. ${ }^{10}$

We restrict our discussion to an isotropic distribution of spherical particles. Short range order is revealed by the structure of the pair correlation function $g(r)$ which is proportional to the probability density of finding a pair of particles separated by a distance $r$. Alternatively, this quantity may be expressed as the autocorrelation function of the number density fluctuations, normalized by the square of the average number density $n$ so that $g(r)$ reaches unity at large separations (independent particles). Pair correlation functions are accessible by scattering experiments where one measures the structure factor

$$
S(q)=1+n \int[g(r)-1] e^{-i \mathbf{q} \cdot \mathbf{r}} d^{3} r
$$

from the normalized measured scattering intensity $I(q)=K \phi a^{6} P(q) S(q)$, where $\phi$ is the particle volume fraction and $a$ is the radius of the particle. $K$ is a constant depending on the optical properties of the particle and the solvent. The quantity $q=|\mathbf{q}|$ is related to the scattering angle $\theta$ by $q=(4 \pi / \lambda) \sin (\theta / 2)$, where $\lambda$ is the wavelength in the suspension. The form factor $P(q)$ which contains the information on the shape and internal structure of the particle is the square of the normalized scattering amplitude $B(q)$. The structure factor $S(q)$ contains the information about the correlations between particles $j$ and $k$ centered at $\mathbf{R}_{j}$ and $\mathbf{R}_{k}$, respectively. Equation (1) can also be written as

$$
S(q)=1+\frac{1}{N} \sum_{j \neq k}^{N}\left\langle e^{i \mathbf{q} \cdot\left(\mathbf{R}_{j}-\mathbf{R}_{k}\right)}\right\rangle,
$$

where the brackets denote the ensemble average and $N$ is the total number of particles. For a system of monodisperse particles interacting through strongly repulsive forces $S(q)$ has a characteristic shape: At low $q$ the structure factor is small due to the low value of the osmotic compressibility $\chi_{\dot{T}}$, since $S(0)=(n / \beta) \chi_{T}$, the quantity $\beta=1 /(k T)$ being the reciprocal thermal energy. At $q \sim 2 \pi n^{1 / 3}$ a characteristic first peak appears. Its position corresponds to the mean interparticle separation where $g(r)$ shows a maximum. At higher $q$ there are less pronounced higher order maxima in $S(q)$ which progressively decay to unity.

According to the ansatz of Ornstein and Zernike ${ }^{11}$ the total correlation function $h(r)=g(r)-1$ between two particles may be split into a part $c(r)$ measuring the direct correlation and an indirect part describing the correlation mediated by the surrounding particles. The direct correlation function $c(r)$ can actually be defined in terms of the total correlation function $h(r)$ by

$$
h(r)=c(r)+n \int h(|\mathbf{r}-\mathbf{s}|) c(s) d^{3} s
$$

The Ornstein-Zernike relation (3) has to be completed with an approximate closure relation which links the correlation functions $h(r)$ or $c(r)$ to the pair interaction potential $u(r)$. The mean-spherical approximation ${ }^{10} c(r)=-\beta u(r)$ has been widely used in the study of micellar suspensions at high volume fractions. ${ }^{4}$ Its popularity lies in the fact that analytical expressions are available for pair correlation functions between macroions (i.e., particles) and electrolyte ions interacting by Coulomb potentials. ${ }^{12,13}$ Yet its applicability is restricted to weakly charged systems since for increasing charge asymmetries the resulting $g(r)$ tend to be negative at small separations. The Percus-Yevick approximation used in the study of hard-sphere systems and the hypernetted-chain approximation ${ }^{10}$ represent a higher degree of sophistication than the mean-spherical approximation, but without the benefits of analytical solutions for charged systems. One way to combine the advantages of both approximations is the empirical Rogers-Young closure ${ }^{14}$

$$
\begin{aligned}
h(r)= & -1+\exp [-\beta u(r)] \\
& \times\left(1+\frac{\exp \{f(r)[h(r)-c(r)]\}-1}{f(r)}\right)
\end{aligned}
$$

which interpolates with the mixing function $f(r)=1-e^{-\alpha r}$ between Percus-Yevick $(\alpha \rightarrow 0)$ and hypernetted chain $(\alpha \rightarrow \infty)$. The mixing parameter $\alpha$ has to be chosen such that the isothermal compressibilities $\chi_{T}^{(f)}$ and $\chi_{T}^{(v)}$ calculated via the fluctuation and the virial route, respectively, coincide. The fluctuation compressibility is directly calculated from 
the structure factor by $\chi_{T}^{(f)}=(\beta / n) S(0)$, while the virial compressibility $\chi_{T}^{(\nu)}=(\partial[\beta P] / \partial n)_{T}^{-1}$ is computed from the equation of state

$$
\frac{\beta P}{n}=1-\frac{2 \pi \beta n}{3} \int_{0}^{\infty} g(r) \frac{d u(r)}{d r} r^{3} d r .
$$

On the level of a primitive model description all the mutual correlations between the macroions and the small electrolyte ions interacting via the bare Coulomb potential have to be considered. If one is interested in the structure of the macroions only, an effective one-component description without the degrees of freedom of the small ions is more appropriate. The screening of the bare Coulomb interaction by the electrolyte ions results in a potential of mean force $u(r)$ between the macroions which depends on the charge and concentration of the macroions. Medina-Noyola and McQuarrie ${ }^{16}$ demonstrated that the mean-spherical approximation in the limit of vanishing macroion concentration $(n \rightarrow 0)$ and electrolyte ions of vanishing size yields the screened Coulomb or Yukawa potential

$$
u(r)=\frac{Z^{2} e_{0}^{2}}{4 \pi \epsilon \epsilon_{0}}\left(\frac{e^{\kappa \alpha}}{1+\kappa a}\right)^{2} \frac{e^{-\kappa r}}{r},
$$

where $e_{0}$ is the charge of the electron and $\kappa=\left(\beta e_{0}^{2} \Sigma_{i} \rho_{i} / \epsilon \epsilon_{0}\right)^{1 / 2}$ is the reciprocal Debye screening length; the sum is taken over the (univalent) counterions and electrolyte ions with bulk number density $\rho_{i}$. The solvent enters the interaction potential by its dielectric constant $\epsilon$ only. The macroions have an effective charge $Z e_{0}$.

Equation (6) is equivalent to the far-field limit $(r \rightarrow \infty)$ of the Derjaguin-Landau-Verwey-Overbeek (DLVO) potential $^{17}$ which was derived for Debye screening lengths which are large compared with the size of the particles (i.e., $\kappa a$ is small). The prefactor $e^{\kappa a} /(1+\kappa a) \equiv X_{0}$ in Eq. (6) assumes, however, that the concentration of added salt exceeds the concentration of the counterions. For low salt concentrations and higher volume fractions an extended justification for the Yukawa form of $u(r)$ for weakly charged macroions has been given in a study by Belloni ${ }^{15}$ using the mean-spherical approximation. The prefactor $X_{0}$ is then replaced by a function $X$ which depends on $\kappa a, Z$, and $n$. In de-ionized suspensions of particles whose radius is large compared with the Bjerrum length $l_{B}=\beta e_{0}^{2} /\left(4 \pi \epsilon \epsilon_{0}\right)$ the relative deviation of the prefactor $X$ from $X_{0}$ does not exceed $10^{-3}$. It is interesting to note that for increasing charge the classical DLVO prefactor $X_{0}$ is reached at smaller concentrations of added salt.

For highly charged systems one has to take into account nonlinear screening by the counterions which is neglected by the mean-spherical approximation. The numerical treatment of the primitive model within the hypernetted-Chain approximation fails to converge for large charge asymmetries. ${ }^{18}$ Very recently, Löwen et al. ${ }^{19}$ have used combined density functional and molecular dynamics simulation techniques to investigate highly asymmetric colloidal suspensions. They found considerable deviations from the DLVO behavior in the macroion pair and triplet structure if the charge $Z$ in the Yukawa potential [cf. Eq. (6)] is identified with the charge appearing in the Coulomb potential mediating the interaction in the primitive model. However, it was found that a pairwise additive Yukawa potential can be fitted to the configurationdependent forces between the particles. ${ }^{20}$ The resulting charges and Debye screening lengths indicate that in the description of concentrated and/or highly interacting systems with the Yukawa potential (6) the parameters $Z$ and $\kappa$ should be considered rather as effective than bare charges and screening parameters, respectively.

Apart from few exceptions, the interpretation of light scattering experiments on real colloidal suspensions is complicated by polydispersity in size, charge, or refractive index. For the practical computation of correlation functions the continuous size distribution is generally replaced by a small number $m$ of components. ${ }^{21}$ The radii $a_{\mu}$ and the number densities $n_{\mu}(\mu=1, \ldots, m)$ of the components are chosen such that the first $2 m$ moments of the histogram distribution coincide with those of the original distribution. As the scattering amplitude is now a weighted sum of the contributions of the individual components, the normalized scattering intensity $I(q)$ no longer factors into a part depending only on single-particle properties and another describing the interparticle correlations. Yet $I(q)$ can be written, in analogy to the monodisperse expression, as $I(q) \propto \vec{P}(q) \bar{S}(q)$. The quantity $\bar{P}(q)$ is the form factor averaged over the size distribution and $\bar{S}(q)$ is the measured structure factor

$$
\bar{S}(q)=\frac{1}{\bar{P}(q)} \sum_{\mu, \nu=1}^{m} B_{\mu}(q) B_{\nu}(q) S_{\mu \nu}(q) .
$$

Note that the measured structure factor contains singleparticle quantities such as the normalized scattering amplitude $B_{\mu}(q)$. The quantities $S_{\mu \nu}$ are the partial structure factors

$$
S_{\mu \nu}(q)=\frac{1}{N} \sum_{j=1}^{N_{\mu}} \sum_{k=1}^{N_{\nu}}\left\langle e^{i \mathbf{q} \cdot\left(\mathbf{R}_{j}^{(\mu)}-\mathbf{R}_{k}^{(\nu)}\right)}\right\rangle,
$$

where $N=\Sigma_{\mu=\mathrm{I}}^{m} N_{\mu}$ and $\mathbf{R}_{j}^{(\mu)}$ is the position of particle $j$ of species $\mu$. For a mixture of $m$ components there are $m(m$ $+1) / 2$ independent partial structure factors $S_{\mu \nu}(q)$, expressing the correlations of the density fluctuations of components $\mu$ and $\nu$. Since particles of different components have different radii $a_{\mu}$ and carry different charges $Z_{\mu}$, the functions $S_{\mu \nu}(q)$ have their maxima at different values of $q$. As a consequence the main peak of $\bar{S}(q)$ is reduced and shifted with increasing polydispersity and the oscillations at higher $q$ are progressively washed out. In addition, the large particles contribute, although their number is small, most of the scattering intensity at low angles. As the scattering amplitude $B_{\mu}(q)$ of the individual spheres is incorporated in $\bar{S}(q)$ an increase in polydispersity results in an increased $\bar{S}(0)$, as has been pointed out by D'Aguanno and Klein. ${ }^{21}$

For moderate polydispersities the interaction potential between particles with radii $a_{\mu}$ and $a_{\nu}$ and effective charges $Z_{\mu}$ and $Z_{\nu}$ is a generalization of Eq. (6)

$$
u_{\mu \nu}(r)=\frac{Z_{\mu} Z_{\nu} e_{0}^{2}}{4 \pi \epsilon \epsilon_{0}} \frac{e^{\kappa a_{\mu}}}{1+\kappa a_{\mu}} \frac{e^{\kappa a_{\nu}}}{1+\kappa a_{\nu}} \frac{e^{-\kappa r}}{r} .
$$




\section{B. Surface chemistry}

The description of the chemical equilibrium in the presence of a charged interface has to account for the inhomogeneous distribution of counterions and electrolyte ions in the vicinity of the surface. In thermal equilibrium the average force on an ion due to its concentration gradient is balanced by the mean force exerted by the electrical field originating from the inhomogeneous charge distribution. This force balance is expressed in terms of the mean electrostatic potential $\Phi(r)$ at a position $\mathbf{r}$ in the electrolyte by the PoissonBoltzmann equation ${ }^{24}$

$$
\nabla^{2} \Phi(\mathbf{r})=\frac{e_{0}}{\epsilon \epsilon_{0}}\left(\rho_{b}^{(-)} e^{e_{0} \beta \Phi(\mathbf{r})}-\rho_{b}^{(+)} e^{-e_{0} \beta \Phi(\mathbf{r})}\right),
$$

where $\rho_{b}^{(+)}$and $\rho_{b}^{(-)}$are the number densities of the cations and anions, respectively, in the electrolyte solution at a distance where the mean electrustatic potential has decayed to zero. Note that the quantities $\rho_{b}^{( \pm)}$are identical to the bulk concentrations only for high salt concentrations, i.e., if the charged surface is immersed in an infinite electrolyte volume. If the Debye screening length $\kappa^{-1}$ is small compared to the particle radius the Poisson-Boltzmann equation reduces to its one-dimensional version for planes. In the limit of high salt concentration (compared to the number of counterions) the Poisson-Boltzmann Eq. (10) simplifies to the GouyChapman form

$$
\frac{d^{2} \Psi}{d x^{2}}=\kappa^{2} \sinh \Psi
$$

where we have introduced the reduced potential $\Psi=\beta e_{0} \Phi$. The application of Gauss' theorem ${ }^{24}$ to the solution of Eq. (11) yields the relationship between the surface charge density $\sigma$ and the electrostatic potential at the surface $\Psi_{0}$

$$
\sigma=\frac{2 \epsilon \epsilon_{0}}{\beta e_{0}} \kappa \sinh \left(\Psi_{0} / 2\right) .
$$

The simple double layer model, based on Eq. (10), requires vanishing ionic size. A first step to include the finite size of the small ions neglected by Eq. (10) is the introduction of a Stern layer. Here the ions are assumed to approach the charged surface not closer than a distance $d$ given by their hydration shell radius. These immobilized ions partially neutralize the surface charge (see Fig. 1). As a consequence the potential drops from $\Psi_{0}$ to the diffuse layer potential $\Psi_{d}$ at the outer Helmholtz plane. This is equivalent to a pair of charged plates with a capacitance per unit area

$$
C_{S}=\beta e_{0} \sigma /\left(\Psi_{0}=\Psi_{d}\right) \text {. }
$$

The charge density outside the outer Helmholtz plane is a diffuse ionic atmosphere which obeys the PoissonBoltzmann equation. If for a finite surface charge density the diffuse layer potential $\Psi_{d}$ approaches $\Psi_{0}$, the Stern layer model reduces to the simple diffuse layer model for $C_{S} \rightarrow \infty$.

Now we have introduced the electrostatical concepts to treat chemical equilibrium. Polymeric latex particles functionalized with weakly acidic carboxylic $(-\mathrm{COOH})$ groups usually carry a small amount of sulfate $\left(-\mathrm{SO}_{4}^{-}\right)$groups (cf. Fig. 2). The strongly acidic sulfate groups are completely
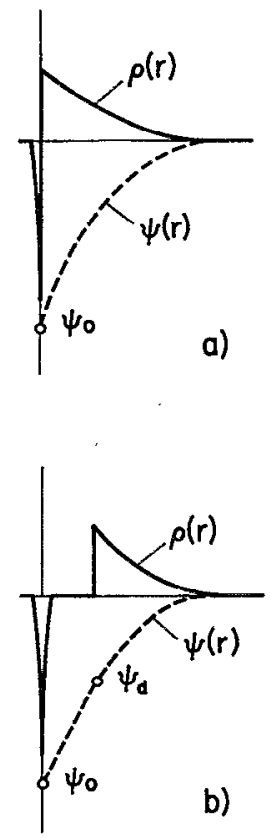

FIG. 1. Diffuse-layer (a) and Stern model (b) of the particle-electrolyte interface. In the diffuse layer model the co- and counterions are allowed direct contact with the particle surface (full line). The electrostatic potential $\Psi(r)$ (dashed line) increases from its value $\Psi_{0}$ at the surface to zero in the bulk. In the Stern model the electrolyte ions are held apart from the charged surface by their finite size. This gives rise to a linear potential increase across the (charge-free) Stern layer from $\Psi_{0}$ to $\Psi_{a}$. Outside the outer Helmholtz plane the elcctrostatic potential is determined by the Boltzmann statistics of the charge distribution $\rho(r)$.

dissociated. The carboxylic groups are in chemical equilibrium with their deprotonated form $\left(-\mathrm{COO}^{-}\right)$by the dissociation reaction

$$
-\mathrm{COOH} \rightleftharpoons-\mathrm{COO}^{-}+\mathrm{H}^{+}
$$

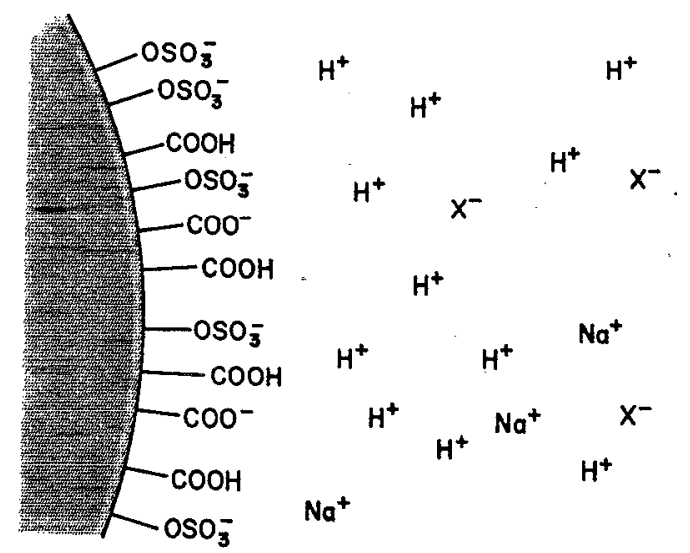

FIG. 2. Schematic representation of a particle surface carrying ionizable carboxylic and sulfate groups. The sulfate groups are strongly acidic and are completely dissociated even when the less acidic carboxylic groups are still fully protonated. The potential-determining protons are allowed to adsorb specifically to the carboxylic groups, whereas the salt ions $\mathrm{Na}^{+}$and $X^{-}$ remain in the diffuse layer. 
which is characterized by the dissociation constant

$$
K_{\mathrm{COOH}}=\frac{\left[-\mathrm{COO}^{-}\right]\left[\mathrm{H}^{+}\right]_{0}}{[-\mathrm{COOH}]},
$$

where the brackets denote volume concentrations and the subscript indicates the concentration of the dissolved protons at the surface.

The aqueous solution around the particle contains, in addition to the potential-determining counterions $\mathrm{H}^{+}$, univalent electrolyte cations $\mathrm{Na}^{+}$, and anions, schematically denoted by $\mathrm{X}^{-}$, which do not undergo specific adsorption reactions with the surface groups. Autodissociation of water releases protons and hydroxide ions according to

$$
\mathrm{H}_{2} \mathrm{O} \rightleftharpoons \mathrm{H}^{+}+\mathrm{OH}^{-} \text {. }
$$

The law of mass action is expressed by the local equilibrium constant

$$
K_{W}=\left[\mathrm{H}^{+}\right]_{b}\left[\mathrm{OH}^{-}\right]_{b},
$$

where the subscript on the right-hand side denotes the bulk concentration. In a titration experiment the addition of the strong base $\mathrm{NaOH}$ increases the surface charge by the neutralization of protons both from the surface and from the solution. The quantity $p \mathrm{H}_{b}=-\log \left[\mathrm{H}^{+}\right]_{b}$ is directly measured as a function of the volume of $\mathrm{NaOH}$ added. To determine the particle charge $Z$ given by

$$
Z=-4 \pi \sigma a^{2} / e_{0}=\frac{N_{A}}{n}\left(\left[-\mathrm{COO}^{-}\right]+\left[-\mathrm{SO}_{4}^{-}\right]\right)
$$

one has to solve Eqs. (15), (17), and (18) for a given value of $p \mathrm{H}_{b}$, together with the mass balances for the surface species and for the protons

$$
\begin{aligned}
& {\left[-\mathrm{COO}^{-}\right]+[-\mathrm{COOH}]=c_{\mathrm{COOH}},} \\
& {\left[-\mathrm{SO}_{4}^{-}\right]=c_{\mathrm{SO}_{4}},} \\
& {\left[\mathrm{H}^{+}\right]_{b}+[-\mathrm{COOH}]-\left[\mathrm{OH}^{-}\right]_{b}=c_{\mathrm{HX}}-c_{\mathrm{NaOH}},}
\end{aligned}
$$

where $c_{\mathrm{COOH}}$ and $c_{\mathrm{SO}_{4}}$ are the total concentrations of ionizable carboxylic and sulfate groups, respectively, and $c_{\mathrm{HX}}$ and $c_{\mathrm{NaOH}}$ are the concentrations of added acid and base, respectively. The surface concentration of protons appearing in Eq. (15) is related to $\left[\mathrm{H}^{+}\right]_{b}$ by the surface potential $\Psi_{0}$

$$
\left[\mathrm{H}^{+}\right]_{0}=\left[\mathrm{H}^{+}\right]_{b} e^{-\Psi_{0}} \text {. }
$$

The solution of the set of equations (12), (15), (17)-(22) then constitutes the chemical dissociation equilibrium in the presence of a charged interface at high salt concentration.

Equation (22) has an important consequence: if the surface potential is strongly negative the proton concentration at the surface may be high even if the bulk solution is quite alkaline. In addition, Eq. (22) shows how the chemical behavior can be disentangled from electrostatics: For a given surface charge or degree of surface dissociation the bulk proton concentration $\left[\mathrm{H}^{+}\right]_{b}$ depends on the ionic strength of the electrolyte; the dissociation constant $K_{\mathrm{COOH}}$, however, does not depend on electrostatics. Thus if we express the surface charge in terms of $p \mathrm{H}_{0}$ rather than in $p \mathrm{H}_{b}$, the charging curves at different ionic strengths coincide onto a master curve $^{26,27}$ if the surface chemistry is described by the exchange of protons only.

Note that in the Stern model the free protons are excluded from the surface so $\left[\mathrm{H}^{+}\right]_{0}$ has to replaced by the proton concentration at the outer Helmholtz plane $\left[\mathrm{H}^{+}\right]_{d}$ $=\left[\mathrm{H}^{+}\right]_{b} e^{-\Psi_{d}}$. Accordingly, the law of mass action Eq. (15) is replaced by

$$
\frac{\left[-\mathrm{COO}^{-}\right]\left[\mathrm{H}^{+}\right]_{d}}{[-\mathrm{COOH}]}=K_{\mathrm{COOH}} e^{\beta \sigma / C_{S}} .
$$

By the presence of the Stern capacitance $C_{S}$ in the effective mass action law (23) the charging curve $\sigma \mathrm{vs} p \mathrm{H}_{d}$ is broadened. This is in marked contrast to the behavior of a monoprotic acid such as acetic acid where the charging curve is a narrow, S-shaped function of $p \mathrm{H}_{0}$.

\section{Poisson-Boltzmann cell model}

In the last section we have presented the GouyChapman model of an isolated charged interface in the presence of surface dissociation as a chemical charge regulation mechanism. This model is strictly valid only in the limit of high salt concentration.

The determination of accurate interaction potentials between macroions requires the study of the full primitive model system, taking into account correlations between the ions. As mentioned in Sec. II A the solution of the primitive model within the mean-spherical approximation (which, as a linear theory, neglects ion correlations) yields the Yukawa potential as a good description of the true interaction potential between two macroions for large distances. The strength of the interaction is given by an effective rather than the bare charge. Therefore the bare charge has to be renormalized in order to obtain the effective charge appearing in the Yukawa potential Eq. (6). Various approaches have been studied in the past. Belloni ${ }^{22}$ studied a highly charged primitive model system within the hypernetted-chain approximation. Effective charges were obtained from fits of one-component structure factors to the macroion structure factors calculated in the primitive model. For too high bare charges, however, the hypernetted-chain approximation was found to produce unphysical results.

Very recently, Fushiki ${ }^{23}$ studied a primitive model system with molecular dynamics simulation. For each macroion configuration in the simulation the Poisson-Boltzmann equation for the surrounding ion atmosphere was solved. The resulting forces on the macroions were then used to generate the next macroion configuration. The effective charge in the Yukawa potential of a one-component system was then adjusted such that the macroion-macroion structure factors coincide. Despite the considerable numerical effort in Fushiki's approach its applicability is limited to relatively small charge asymmetries, albeit larger than the hypernetted-chain integral equation allows to treat.

The cell model first studied by Alexander et al. ${ }^{8}$ presents a method for charge renormalization which is computationally simpler since the complicated many-body system is replaced by an effective one-particle system. A single macro- 

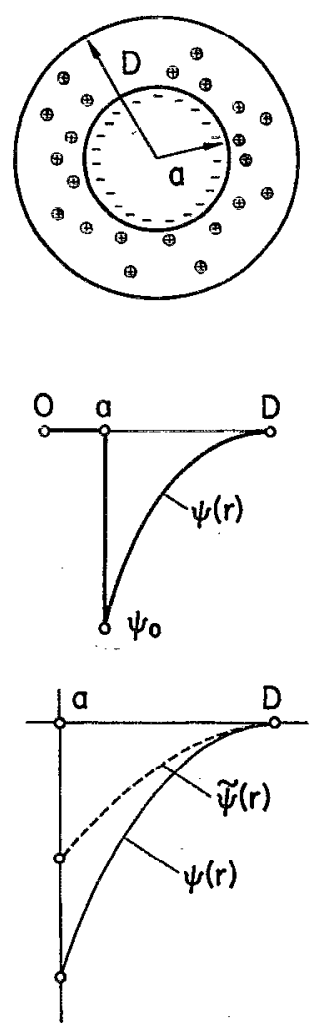

FIG. 3. Spherically symmetric Wigner-Seitz cell used in the PoissonBoltzmann cell model (top). The particle of radius $a$ is surrounded by a spherically symmetrical charge density distribution. The radius $D$ of the cell is chosen such that the particle volume fraction is obtained. Since the cell is electroneutral as a whole, $d \Phi / d r$ vanishes at the cell boundary. The potential at the boundary $\Phi(D)$ can be arbitrarily chosen (middle part of the figure); in our calculations we use the convention $\Phi(D)=0$. Charge renormalization is illustrated in the bottom part of the figure. In the vicinity of the cell boundary the exact Poisson-Boltzmann potential $\Psi(r)$ is matched with the solution $\tilde{\Psi}(r)$ of the linearized Poisson-Boltzmann equation such that the two potentials coincide in magnitude and slope. The derivative of $\tilde{\Psi}(r)$ at the particle surface (which is proportional to the effective charge) is smaller in magnitude than the slope of the cxact Poisson-Boltzmann potential (which is proportional to the bare charge $Z$ ).

ion is fixed at the center of a spherical Wigner-Seitz cell (cf. Fig. 3). The presence of the surrounding macroions is accounted for by the finite size of the cell $D=a \phi^{-1 / 3}$, where $\phi$ is the particle volume fraction and $a$ is an effective particle radius given by $a=R^{3} / R^{2}$, the quantities $\overline{R^{2}}$ and $R^{3}$ being the second and third moments of the particle size distribution.

For any type of charge regulation mechanism, a discussion of the cell model has to distinguish between three cases: the high salt regime (which has been discussed above), the intermediate, and the low salt regime.

For low salt concentration only the counterions contribute to the charge density. Equation (10) then simplifies to

$$
\frac{d^{2} \Psi}{d r^{2}}+\frac{2}{r} \frac{d \Psi}{d r}=-\frac{\beta e_{0}^{2}}{\epsilon \epsilon_{0}} \rho_{D}^{(+)} e^{-\Psi}
$$

where $\rho_{D}^{(+)}$is the counterion number density at the cell boundary at $r=D$. The two boundary conditions for the solution of Eq. (24) are

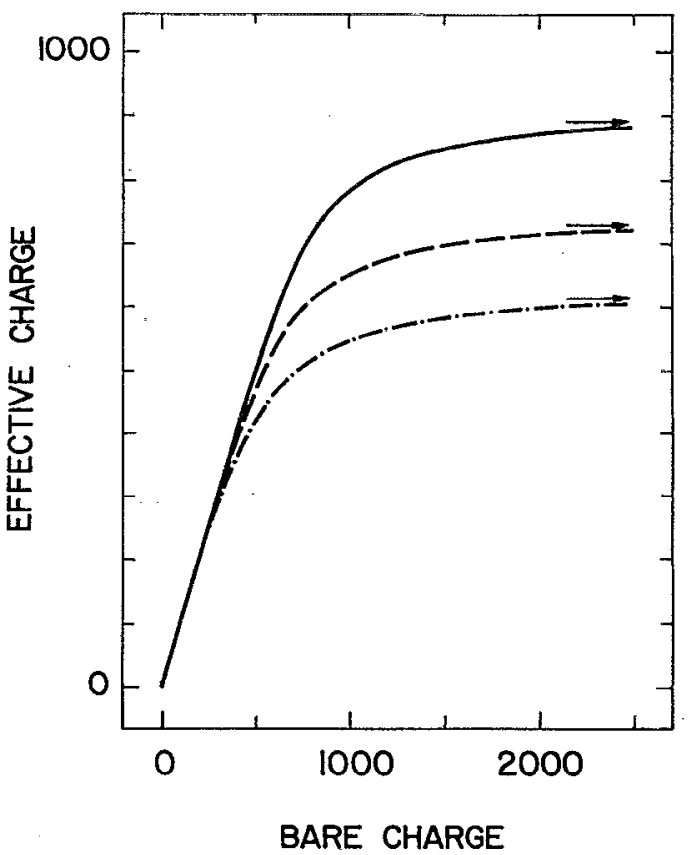

ГTG. 4. Effective charge $\tilde{Z}$ as a function of the bare charge $Z$ for volume fractions $\phi=10^{-5}$ (full line), $10^{-4}$ (dashed line), and $10^{-3}$ (dash-dotted line) in the spherical cell model for $a=51.3 \mathrm{~nm}$ particles and Bjerrum length $l_{B}=0.72 \mathrm{rm}$. At high bare charges the effective charge converges to a saturation value (indicated by the arrow).

$$
\Psi(D)=0
$$

since only differences in the electrostatic potential are important, and the condition for global electroneutrality

$$
d \Psi /\left.d r\right|_{r=D}=0 \text {. }
$$

Equation (24) is integrated at $r=a$ for a fixed value of $\rho_{D}^{(+)}$. The surface charge density $\sigma$ is then calculated from $\sigma=-\left[\epsilon \epsilon_{0} /\left(\beta e_{0}\right)\right] d \Psi /\left.d r\right|_{r=a}$. As the surface charge is successively increased, the counterion concentration at the cell boundary, however, does not exceed a saturation value. In order to maintain overall electroneutrality the counterion concentration profile in the immediate vicinity of the particle surface decreases steeply, i.e., the counterions appear to accumulate on the particle surface. At the cell boundary the potential $\Psi(r)$ is asymptotically approximated by the solution of the linearized Poisson-Boltzmann equation

$$
\frac{d^{2} \tilde{\Psi}}{d r^{2}}+\frac{2}{r} \frac{d \tilde{\Psi}}{d r}=-\frac{\beta e_{0}^{2}}{\epsilon \epsilon_{0}} \rho_{D}^{(+)}(1-\tilde{\Psi}) .
$$

Equation (27) with the boundary conditions of Eqs. (25) and (26) has an analytical solution of the form $\tilde{\Psi}(r)$ $=1+\tilde{\Psi}_{0}\left(A_{-} e^{-\tilde{\kappa} r}+A_{+} e^{\tilde{\kappa} r}\right) / r$ where $A_{ \pm}$are constants depending on $\rho_{D}^{(+)}$and $\phi$. At small distances from the surface, however, $\tilde{\Psi}(r)$ underestimates the magnitude and slope of $\Psi(r)$. Thus the surface charge density $\tilde{\sigma}=\left[\beta \epsilon \epsilon_{0}\right]$ $\left.\left(e_{0} a\right)\right](\tilde{\kappa} a+1) \tilde{\Psi}_{0}$ and the screening parameter $\tilde{\kappa}=\left(4 \pi l_{B} \rho_{D}^{(+)}\right)^{1 / 2}$ have to be interpreted as renormalized effective quantities. Figure 4 illustrates the dependence of the effective charge $\tilde{Z}=-4 \pi a^{2} \tilde{\sigma} / e_{0}$ on the bare charge $Z$. At 
low charges the two quantities are equal. As $Z$ is increased, however, the effective charge saturates to a value $\tilde{Z}_{\infty}$, indicating the onset of counterion condensation.

The intermediate case where the concentrations of counterions and electrolyte ions are of the same order of magnitude is treated in an analogous way. Now the PoissonBoltzmann equation reads

$$
\frac{d^{2} \Psi}{d r^{2}}+\frac{2}{r} \frac{d \Psi}{d r}=\frac{\beta e_{0}^{2}}{\epsilon \epsilon_{0}}\left(\rho_{D}^{(-)} e^{\Psi}-\rho_{D}^{(+)} e^{-\Psi}\right) .
$$

Note that the concentrations of $\rho_{D}^{( \pm)}$of the anions $(-)$and cations $(+)$ at the cell boundary are two free parameters which determine the solution of Eq. (28). They are related by

$$
\rho_{D}^{(+)} \int_{a}^{D} e^{-\Psi(r)} r^{2} d r-Z=\rho_{D}^{(-)} \int_{a}^{D} e^{+\Psi(r)} r^{2} d r,
$$

where the left-hand side is the difference of the total number of cations and the number of counterions. The boundary conditions are again given by Eqs. (25) and (26). Effective charges and screening parameters $\tilde{Z}$ and $\tilde{\kappa}$, respectively, are obtained from the solution of the linearization of Eq. (28).

So far the surface charge has been treated as a free parameter in the cell model independent on the composition of the electrolyte solution around the particle. This situation is realized in suspensions of particles carrying strongly acidic sulfate groups. In the presence of weakly acidic carboxylic groups the surface charge is regulated by the dissociation equilibrium (14). In this case the Poisson-Boltzmann Eq. (28) is solved numerically with

$$
\begin{aligned}
& \rho_{D}^{(+)}=\left[\mathrm{H}^{+}\right]_{D}+\left[\mathrm{Na}^{+}\right]_{D}, \\
& \rho_{D}^{(-)}=\left[\mathrm{OH}^{-}\right]_{D}+\left[\mathrm{X}^{-}\right]_{D}, \\
& K_{W}=\left[\mathrm{H}^{+}\right]_{D}\left[\mathrm{OH}^{-}\right]_{D},
\end{aligned}
$$

such that the mass action law Fq. (15) is fulfilled. As the total number of protons and charges is conserved only globally in the cell (but not on its boundary), the bulk concentrations of the free species in the charge balance Eq. (21) are replaced by the respective volume averaged concentrations

$$
\begin{aligned}
& {\left[\mathrm{H}^{+}\right]_{t}=\eta^{(+)}\left[\mathrm{H}^{+}\right]_{D},} \\
& {\left[\mathrm{Na}^{+}\right]_{t}=\eta^{(+)}\left[\mathrm{Na}^{+}\right]_{D},} \\
& {\left[\mathrm{OH}^{-}\right]_{t}=\eta^{(-)}\left[\mathrm{OH}^{-}\right]_{D},} \\
& {\left[\mathrm{X}^{-}\right]_{t}=\eta^{(-)}\left[\mathrm{X}^{-}\right]_{D},}
\end{aligned}
$$

where $\eta^{( \pm)}=\int_{a}^{D} r^{2} e^{\mp \Psi(r)} d r / \int_{a}^{D} r^{2} d r$. The effective charge $\tilde{Z}$ and Debye screening parameter $\tilde{\kappa}$ are then recovered in the usual way hy solving the linearized Eq. (28). A similar scheme has recently been proposed by Zwetsloot and Leyte. $^{25}$

\section{EXPERIMENT}

\section{A. Particle characterization}

Polystyrene latex spheres synthesized by emulsion copolymerization were purchased in aqueous suspension from Polyscience Inc. (Warrington PA). The weight content of sol-

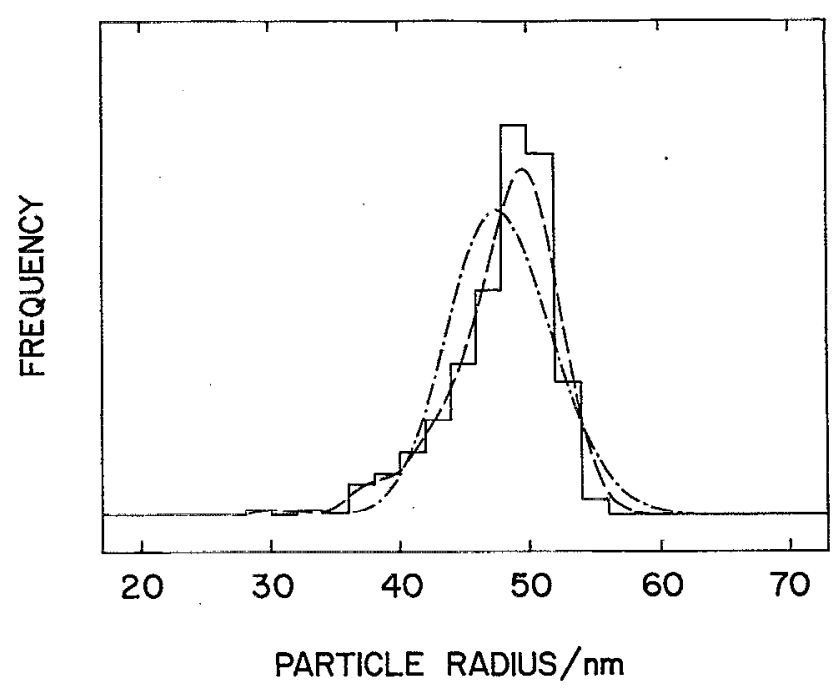

FIG. 5. Particle size distribution obtained by transmission electron microscopy (full line: histogram; dashed line: smoothed distribution calculated from the histogram) and Schultz distribution calculated with the measured average radius $\bar{R}=47.9 \mathrm{~nm}$ and polydispersity $p_{\text {TEM }}=8.4 \%$ (dash-dotted line). Note the tail of the measured distribution at $R<R$ which is in contrast to the tail of the Schultz distribution at $R>\check{R}$.

ids was found to be $2.50 \% \pm 0.01 \%$ using a Perkin-Elmer thermogravimetric analysis instrument. Density of the polystyrene particles was determined in a Nicodenz (Nygaard) density gradient ( $42 \mathrm{~h}$ at $34000 \mathrm{rpm}$ in a Bcckmann model $\mathrm{E}$ analytical ultracentrifuge at $22{ }^{\circ} \mathrm{C}$ ) to be $1.043 \pm 0.002 \mathrm{~g} / \mathrm{cm}^{3}$.

Particle size distribution was analyzed from transmission electron micrographs. They were calibrated using a Pt grid with 2160 lines $/ \mathrm{mm}$. We also analyzed a size distribution from a sample which, after freezing, had been shaded with a thin carbon film. We did not find any significant difference between the shaded and the unshaded size distributions. Figure 5 shows the histogram and the smoothed distribution from a sample of 582 shaded particles. The average radius $\bar{R}$ is $47.9 \pm 0.3 \mathrm{~nm}$ and the normalized variance $p_{\text {TEM }}$ $=\left(\overline{R^{2}} / \bar{R}^{2}-1\right)^{1 / 2}$ is $8.4 \% \pm 0.5 \%$. The measured size distribution shows, unlike the Schultz distribution calculated with the same $\bar{R}$ and $p_{\mathrm{TEM}}$, a characteristic tail at smaller sizes.

Light scattering experiments were performed using a Malvern 4700 goniometer with an $\mathrm{Ar}^{+}$laser (Coherent Innova 200-10) at a wavelength $\lambda_{0}=488 \mathrm{~nm}$. The incident primary beam was vertically polarized and the intensity was adjusted with a combination of a neutral density filter, a halfwave plate, and a polarizer in order to achieve maximum signal amplitude within the linear range of the photomultiplier response.

An apparent hydrodynamic radius was measured with quasielastic light scattering using a Malvern 7032 correlator at angles $10^{\circ} \leqslant \theta \leqslant 120^{\circ}$. At each angle, 10 intensity autocorrelation functions $g^{(2)}(\tau)$ of dilute suspensions with volume fractions ranging from $\phi=1.2 \times 10^{-5}$ to $\phi=3.6 \times 10^{-5}$ in 10 $\mathrm{mM} \mathrm{NaCl}$ were recorded as a function of the correlator lag time $\tau$. We determined the average hydrodynamic radius $\overline{R_{h}}$ from the second-order cumulant expansion of the field autocorrelation function $g^{(1)}(\tau)$ (see Table I). As the computation 
of $g^{(1)}(\tau)$ from $g^{(2)}(\tau)$ with an ill-determined baseline often results in a spurious negative second cumulant, we deter-

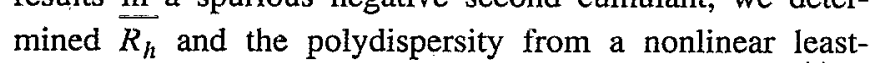
squares fit of the second-order cumulant expansion of $g^{(2)}(\tau)$ with adjustable baseline. The value for the hydrodynamic radius agrees well with the one from the fit of $g^{(1)}(\tau)$, but the polydispersity is grossly overestimated, as also reported by other authors. ${ }^{36}$

Static light scattering with dilute suspensions of volume fraction $\phi=3.6 \times 10^{-5}$ and $\mathrm{NaCl}$ concentration of $10 \mathrm{mM}$ at angles $30^{\circ} \leqslant \theta \leqslant 140^{\circ}$ yielded the angular dependence of the mean scattering intensity $I(q)$. Average intensities were obtained from ten individual measurements at each angle and corrected for variations in the scattering volume by a multiplication with $\sin \theta$. The measurements were repeated at different positions of the cuvette in order to detect systematic errors originating from scratches in the cuvette walls. Data were analyzed using the Guinier approximation for polydisperse homogeneous spheres ${ }^{31}$

$$
\ln I(q)=\ln I_{0}-q^{2} \overline{R_{g}^{2}} / 3
$$

with $I_{0}$ the scattering intensity at $q=0$. The average radius of gyration $\overline{R_{g}}$ was determined from the slope of the Guinier plot.

We measured the specific surface area on previously dialyzed and freeze-dried latex samples by $\mathrm{N}_{2}$ gas adsorption on a Micromeritics Gemini 2360. The specific surface area was determined by fitting the Brunauer-Emmett-Teller adsorption isotherm ${ }^{32}$ to the experimental data points.

Residual traces of cations in the latex particles were determined with atomic absorption spectroscopy on a Varian 400 spectrometer. The total concentrations of residual Na was in the range of the experimental error of $10^{-2} \mathrm{mM}$, corresponding to a maximum of $1.2 \times 10^{4} \mathrm{Na}^{+}$ions per latex particle. This is about $6.5 \%$ of the total number of dissociable surface charges.

\section{B. Structure factor determination}

Static light scattering measurements were performed at $25^{\circ} \mathrm{C}$ with the setup as described above. Prior to the measurements, the samples were diluted from the dialyzed stock suspension and filtered through $1.2 \mu \mathrm{m}$ prerinsed cellulose filters into cylindrical quartz glass cuvettes (Hellma) with a diameter of $10 \mathrm{~mm}$. In order to achieve maximal deionization, the samples were kept for at least 10 days over equal volumes of mixed bed ion exchanger resin (Serdolit MB, Serva) previously washed with deionized water. For one sample we repeated the measurements after purging with Ar in order to remove residual traces of atmospheric carbon dioxide. We found good agreement between the structure factors measured with and without Ar purging.

Scattering intensities were measured in an angular range from $10^{\circ}$ to $140^{\circ}$ in steps varying from $0.2^{\circ}$ at angles around the first intensity maximum to $2.0^{\circ}$ at higher angles. Average intensities were obtained from ten individual measurements at each angle. The measurements were repeated at different positions of the cuvette so that systematic errors duc to scattering from scratches on the glass surface could be detected.
The accessible range of particle volume fractions is limited at low concentrations by the goniometer constraints and the imperfections of the cuvettes at low scattering angles, whereas at higher concentrations deionized samples crystallize, and bright Bragg reflections are superposed to the scattering from the coexisting liquid phase. Although the crystallites can be shear melted by. tumbling the sample, the crystalline structure rearranges within less than $10 \mathrm{~s}$. This time is too short for the transient liquidlike scattering pattern to be measured with our goniometer setup.

\section{Titration experiments}

Titration experiments were carried out with a Schott TR600 system with Metrohm components at $25^{\circ} \mathrm{C}$ in a 12 $\mathrm{ml}$ sample cell. ${ }^{27}$ Electrodes were calibrated with blank titrations. The sample cell was flushed with water-saturated $\mathrm{N}_{2}$ that had been purified by bubbling through concentrated $\mathrm{NaOH}$. The latex samples which had been de-ionized were diluted into a $\mathrm{NaClO}_{4}$ (Merck) electrolyte solution to volume fractions of about $0.5 \%$. Prior to the measurement, the particles were protonated by addition of $\mathrm{HClO}_{4}$ (Merck). In spite of the full neutralization of the carboxylic surface charges the particles did not aggregate. This is possibly due to the presence of negatively charged sulfate groups which are the fragments of the initiator (potassium persulfate) used in the polymerization of the latex. As titration agent we used carbonate-free $\mathrm{NaOH}$ of about the same ionic strength as the background electrolyte. Upon addition of $\mathrm{NaOH}$ the suspension was allowed to equilibrate for $10 \mathrm{~min}$; after that time the $\mathrm{pH}$ of the suspension was measured. Bare particle charges were determined using the law of mass action Eq. (15) assuming that each equivalent of $\mathrm{NaOH}$ added converts an equivalent of carboxylic surface groups to its deprotonated form. Together with the given number of sulfate groups per particle the surface charge density was calculated using Eq. (18).

\section{RESULTS AND DISCUSSION}

\section{A. Particle characterization}

From the transmission electron micrographs the quantities measured in light scattering are calculated from the moments of the size distribution. The radius of gyration is given by $\overline{R_{g}}=\left[3 \overline{R^{8}} /\left(5 \overline{R^{6}}\right)\right]^{1 / 2}$ and for the hydrodynamic radius one uses the relation $\overline{R_{h}}=\overrightarrow{R^{6}} / \overline{R^{5}}$. Compared with the $\overline{R_{h}}$ and $\overline{R_{g}}$ measured by light scattering the corresponding quantities calculated from the size distribution showed to be consistently lower by $5 \%-6 \%$. We attribute this observation either to a systematic error in the calibration of the electron micrographs or to shrinkage of the particles caused by the exposition to the electron beam. This phenomenon has also been reported by other authors. ${ }^{33}$ This is further supported by the observation that the surface area measurements are compatible with the light scattering results. ${ }^{34}$ Based on the assumption that electron microscopy systematically underestimates the particle size, we shifted the size distribution shown in Fig. 5 by $5.5 \%$. Excellent agreement can then be found for both $\overline{R_{g}}$ and $\overline{R_{h}}$. This resulted in values of the average radius 
TABLE I. Summary of the characterization of the polystyrene latex particlcs. In the first column the number average particle radius $\vec{R}$, the polydispersity $p_{\mathrm{TEM}}$, the radius of gyration $\overline{R_{g}}$, the hydrodynamic radius $\overline{R_{h}}$, and the specific surface are a $S$ calculated from the uncorrected size distribution obtained from the transmission electron micrographs are shown. The second column contains the same quantities calculated from the size distribution corrected so that the best agreement with the independently measured quantities from light scattering and $\mathrm{N}_{2}$ gas adsorption experiments (third column) was reached (see the text for details).

\begin{tabular}{lccc}
\hline \hline & $\begin{array}{c}\text { Uncorrected size } \\
\text { distribution }\end{array}$ & $\begin{array}{c}\text { Corrected size } \\
\text { distribution }\end{array}$ & $\begin{array}{c}\text { Independently } \\
\text { measured }\end{array}$ \\
\hline$R / \mathrm{nm}$ & $47.9 \pm 0.3$ & $50.7 \pm 0.3$ & $\ldots$ \\
$p_{\mathrm{TEM}} / \%$ & $8.4 \pm 0.5$ & $8.4 \pm 0.5$ & $\ldots$ \\
$R_{\mathrm{g}} / \mathrm{nm}$ & 38.2 & 40.5 & $40.7 \pm 0.6$ \\
$R_{h} / \mathrm{nm}$ & 49.3 & 52.0 & $51.8 \pm 0.5$ \\
$S /\left(\mathrm{m}^{2} / \mathrm{g}\right)$ & 58.8 & 56.1 & $56.8 \pm 0.2$ \\
\hline \hline
\end{tabular}

and polydispersity of $\bar{R}=50.7 \pm 0.3 \mathrm{~nm}$ and polydispersity $p_{\mathrm{TEM}}=8.4 \% \pm 0.5 \%$. Table I shows a comparison of the independently measured quantities with those calculated from the shifted size distribution.

\section{B. Charging curves}

From the titration data the charging curves were obtained from the difference of titration curves from blank and sample measurements, respectively. The electrolyte conccntrations ranging from 1 to $500 \mathrm{mM}$ were high enough $(\kappa a \geqslant 5$ ) so that the ionic strength was dominated by the background electrolyte and the added acid and base solutions. The surface potentials calculated by Eq. (12) were found to lie in the range from -25 to $-175 \mathrm{mV}$. Figure 6 shows the titration curves with the surface charge density $\sigma$ plotted against the $p \mathrm{H}$ in the bulk for $\mathrm{NaClO}_{4}$ concentrations of $1,10,100$, and $500 \mathrm{mM}$. The charging curves were reproducible to within $10 \%$. The number of dissociated sites is

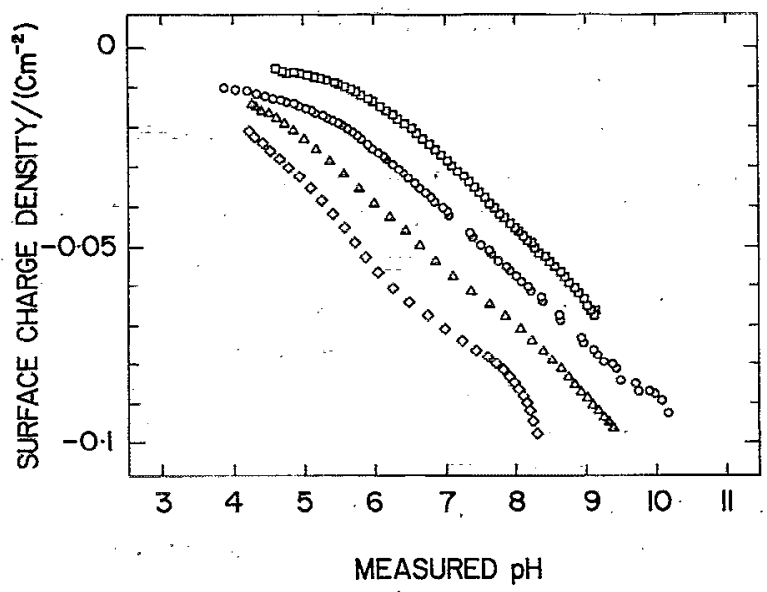

FIG. 6. Titration curves for carboxylate polystyrene latex with average radius $\bar{R}=50.7 \mathrm{~nm}$ and polydispersity $8.4 \%$. The concentrations of the $\mathrm{NaClO}_{4}$ background electrolyte are $1 \mathrm{mM}$ (squares), $10 \mathrm{mM}$ (circles), 100 $\mathrm{mM}$ (triangles), and $500 \mathrm{mM}$ (diamonds). Note the ionic strength dependence which results from the increased screening of the surface charge by the electrolyte. seen to increase linearly with $p \mathrm{H}_{b}$ up to values of about $2.5 \times 10^{4}$. The plateau where all carboxylic groups arc dissociated is not reached in our experiments.

The strong dependence of the charging curves on ionic strength can be understood as follows: for a given particle charge the weak screening at low ionic strength causes protons to be strongly attracted to the surface, resulting in a depletion of protons in the bulk electrolyte. We can eliminate the ionic strength dependence of the surface charge density by expressing $\sigma$ in terms of the $p \mathrm{H}$ at the surface [cf. Eq. (22)]. As described in Sec. II B it should then be possible to construct a universal master curve which characterizes the surface dissociation equilibrium. In order to explain the detailed shape of the master curve, a specific model of the particle surface is needed. In particular we have to specify the number of ionizable groups, their dissociation constant, and the Stern capacitance. We have analyzed our titration data using two different models. In the mixed sulfatecarboxylate model we assumed that the surface contains $5 \%$ sulfate groups in addition to the $4 \times 10^{4}$ carboxylic groups per particle. This numbers given by the producer correspond to a total surface charge density $\sigma_{t}=-0.20 \mathrm{C} / \mathrm{m}^{2}$. Figure 7 shows a fit of the mixed sulfate-carboxylate model to the titration data. At each titration step the surface $p \mathrm{H}$ was calculated from the surface charge density $\sigma$ and the measured $p \mathrm{H}$ in the bulk using Eqs. (22) and (12). The resulting master curve shows a nonzero surface charge density at low $p \mathrm{H}$ which reflects the presence of the strongly acidic sulfate groups. We found a $p \mathrm{~K}$ of the carboxylic groups $p \mathrm{~K}_{\mathrm{COOH}}=4.70$ and a Stern capacitance $C_{S}=0.51 \mathrm{~F} / \mathrm{m}^{2}$.

In order to test the sensitivity of our model calculations on the chemical composition of the surface, we have also analyzed our titration data with a model which assumes carboxylic groups to be present only. The master curve (Fig. 7) starts at a surface $p \mathrm{H}$ of 2 with fully protonated particles. After an increase linear with $p \mathrm{H}_{0}$, the surface charge shows the onset to saturation at $p \mathrm{H}_{0}=9$ which corresponds to the totally ionized state. The best fit to the titration data yields a total surface charge density $\sigma_{t}=-0.11 \mathrm{C} / \mathrm{m}^{2}$, a $p \mathrm{~K}$ for the carboxylic groups $p \mathrm{~K}_{\mathrm{COOH}}=4.30$, and a Stern capacitance $C_{S}=0.58 \mathrm{~F} / \mathrm{m}^{2}$. As the plateau of complete surface dissociation in the accessible $p \mathrm{H}$ range is not reached, both models fit the data equally well with rather different numbers of ionizable surface groups. Thus we cannot definitively determine the total number $\sigma_{t}$ of dissociable surface groups with our titration experiments. In both models the titration curves have to be described with a Stern capacitance in order to account for the broadening of the charging curve.

The physical interpretation of the Stern capacitance is still an open question. Commonly, a Stern model is used to incorporate finite size effects of the electrolyte ions. ${ }^{3}$ An alternative interpretation is to attribute the broadening of the charging curve to strong interaction between neighboring surface groups. In the context of polyelectrolytes, Hill ${ }^{37}$ pointed out that the first-order treatment of nearest-neighbor interactions between surface groups within the Ising model is formally equivalent to a Stern layer description of the proton adsorption isotherm according to Eq. (23). 


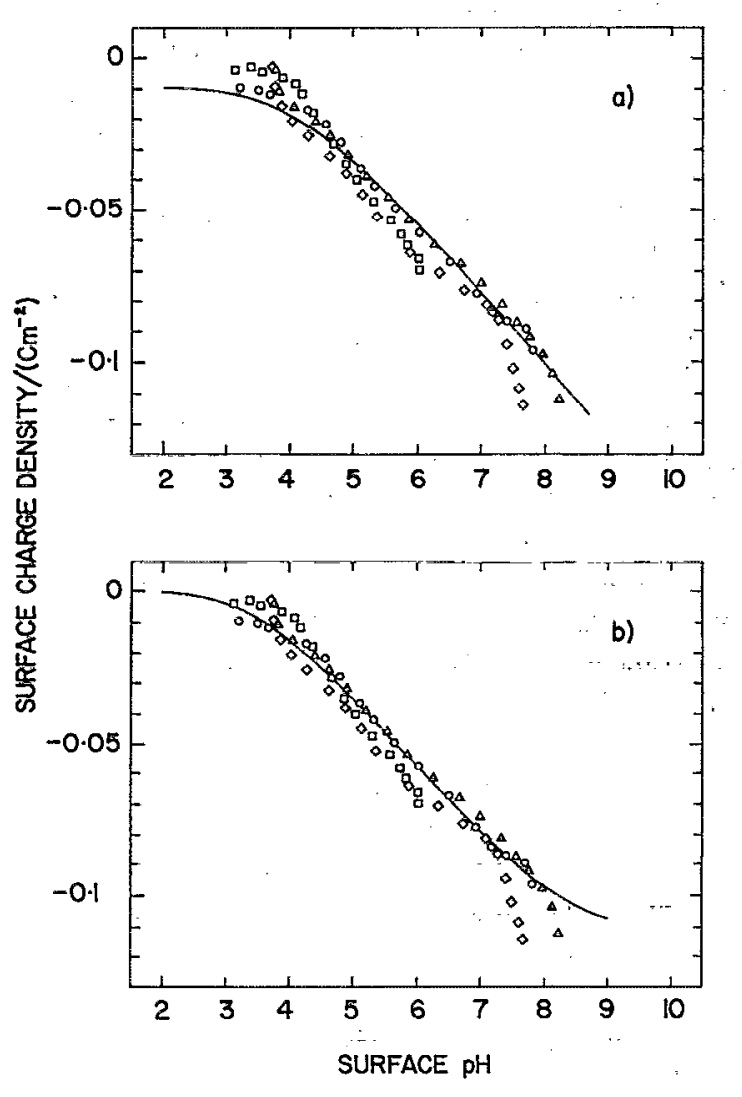

FIG. 7. Master curves for the titration data from Fig. 6. The surface charge density is plotted as a function of the surface $p \mathrm{H}$. For the mixed sulfatecarboxylate model (a) the best fit parameters for the surface dissociation constant and the Stern capacitance are $p \mathrm{~K}_{\mathrm{COOH}}=4.70$ and $C_{S}=0.51 \mathrm{~F} / \mathrm{m}^{2}$, assuming a total density of ionizable surface groups $\sigma_{t}=-0.20 \mathrm{C} / \mathrm{m}^{2}$ with $5 \%$ sulfate groups. The pure carboxylate model (b) yields as best fit parameters $p \mathrm{~K}_{\mathrm{COOH}}=4.30, C_{S}=0.58 \mathrm{~F} / \mathrm{m}^{2}$, and $\sigma_{t}=-0.11 \mathrm{C} / \mathrm{m}^{2}$. Note that the pure model contains a total number of charges which is about half the value assumed in the mixed model. The broadening in the master curves results from the presence of a Stern capacitance.

\section{Structure factors}

The polydisperse structure factor $\bar{S}(q)$ was extracted from the average scattering intensity by normalizing $I(q)$ with the average particle form factor $\bar{P}(q)$. In principle one could directly use a measurement of $I(q)$ with a dilute noninteracting sample for this normalization. However, precise measurements at low values of $q$ are much more difficult for very dilute suspensions and lead to considerable errors in the range of scattering vectors where we expect to observe a peak in the structure factor (cf. Fig. 8). Therefore, we preferred normalization with the average form factor calculated by the Guinier approximation $\mathrm{Eq}$. (37) using the experimentally determined $R_{g}=40.7 \mathrm{~nm}$ (cf. Table I). Typical examples of $\bar{S}(q)$ at different volume fractions are shown in Fig. 9. After normalization the oscillations in the structure factor at angles above $120^{\circ}$ were slightly distorted. We attribute this observation to some multiple scattering from the sample. As shown by Dhont, ${ }^{38}$ the contribution of double scattering for low volume fractions increases with scattering angle and passes through a maximum around $\theta=120^{\circ}$. Thus

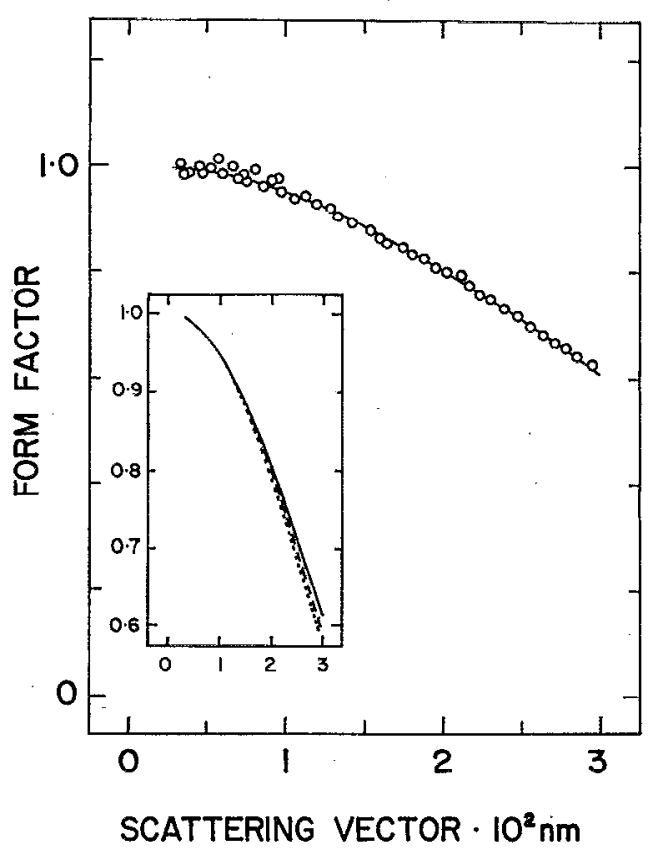

FIG. 8. Measured particle form factor (circles) and best fit calculation using the Guinier approximation Eq. (37) with $\overline{R_{g}}=40.7 \mathrm{~nm}$. In the inset the Guinier approximation is compared with the polydisperse form factors calculated with Mic theory (homogencous sphercs with complex index of refraction $r=1.591+i 0.001)$. The dotted line is the prediction for a Schultz distribution with $\bar{R}=50.7 \mathrm{~nm}$ and $8.4 \%$ polydispersity; the dashed line is calculated using the size distribution determined from the electron micrographs.

the widely used method of obtaining structure factors by normalizing the reduced scattering intensity $I(q) / \bar{P}(q)$ with its value at the highest accessible $q$ may lead to arbitrary shifts in $\bar{S}(q)$ which propagate into erroneous effective charges. A useful and reliable way of normalizing scattering intensities is the estimation of their asymptotic value at large scattering vectors $q$ by a Padé type method used to accelerate convergence in sequences. This type of baseline estimation uses the information in the oscillating part of $I(q) / \bar{P}(q)$ at lower scattering angles $\left(\theta \leqslant 100^{\circ}\right)$ where multiple scattering is negligible. Using the NAG implementation of Shanks' algorithm ${ }^{39,40}$ we found that the method is stable even if the slowly diverging distortion at high $q$ is included in the baseline estimation.

Volume fractions and effective charges were determined by fitting the position and the height, respectively, of the first peak in the calculated $\bar{S}(q)$ to the experimental scattering data. Polydisperse structure factors were calculated with the hypernetted-chain closure for a three-component size distribution with the average particle radius $\bar{R}=50.7 \mathrm{~nm}$ and the polydispersity $p_{\text {TEM }}=8.4 \%$ measured with transmission electron microscopy. We found volume fractions ranging from 2.11 to $9.18 \times 10^{-4}$. These values are smaller than the volume fractions calculated from the experimental dilution factor by $48 \%$ for the lower concentrations and $8 \%$ at the higher concentrations. We attribute this observation to adsorption of the latex particles onto the surface of the ion exchange resin. For the volume fractions $2 \times 10^{-4} \leqslant \phi \leqslant 7 \times 10^{-4}$ we find effective 

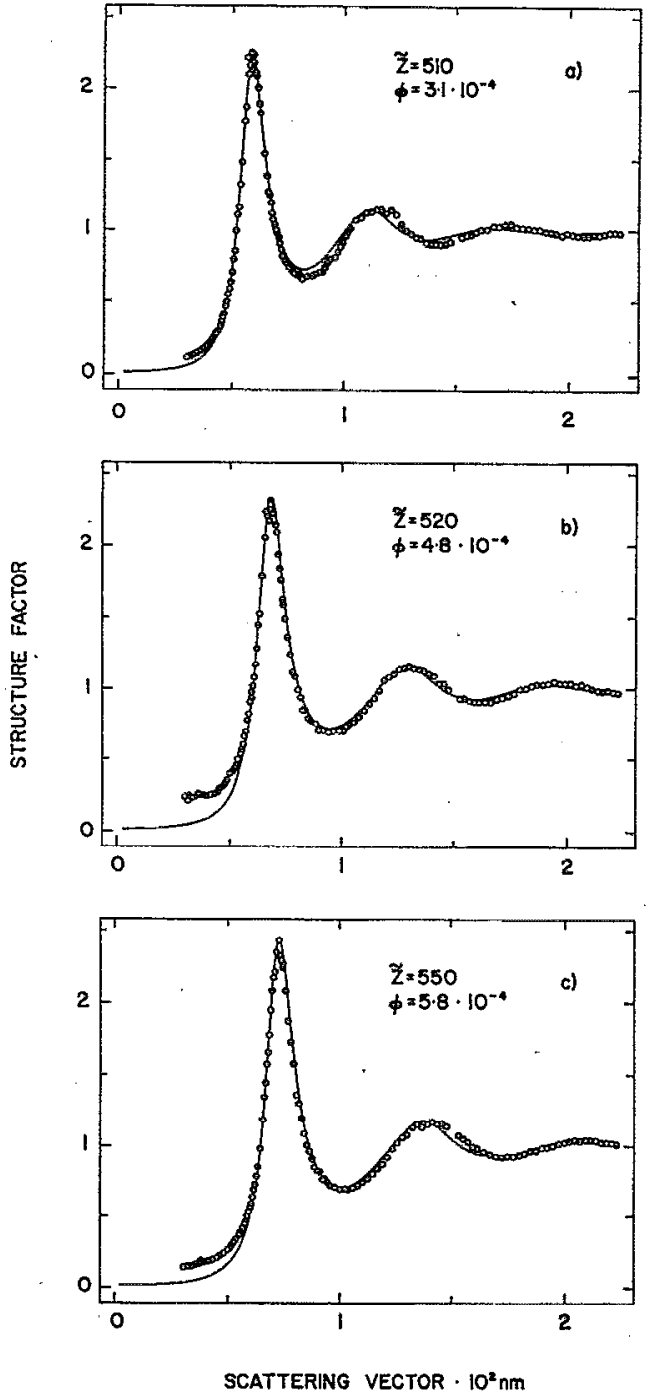

FIG. 9. Measured structure factors (circles) and polydisperse calculation with the polydisperse hypernetted-chain closure (full line). For the calculation, the Schultz distribution for the particle radii was replaced by an equivalent histogram distribution with three components. The mean particle radius $\bar{R}=50.7 \mathrm{~nm}$ and the polydispersity of $8.4 \%$ were taken from the experimentally determined size distribution.

charges which cluster around $\tilde{Z} \sim 560$. For these effective charges and volume fractions $\kappa a \leqslant 0.1$, and the deviation of Belloni's prefactor $X$ in the Yukawa potential (6) from the DLVO expression $e^{\kappa a} /(1+\kappa a)$ is in the order of $10^{-4}$. Without any further adjustment of the polydispersity the experimental $\bar{S}(q)$ is in good quantitative agreement with the calculation (cf. Fig. 9). The measured scattering intensities at low values of $q$, however, are underestimated by the theoretical prediction by almost one order of magnitude. This discrepancy could possibly be due to incomplete removal of aggregates which preferentially scatter into the forward direction. An estimation of the errors in the fitted volume fractions and fitted effective charges was obtained by repeating the fitting procedure for several independently prepared and measured samples at each volume fraction. The volume frac- tions were reproducible to within $10 \%$, whereas the effective charges were afflicted by relative errors up to $24 \%$ at the lowest volume fractions. This indicates that in some of the samples deionization may not have been complete.

The effective charges from our experiments (cf. Fig. 10) are comparable with the results of Versmold et $\mathrm{al}^{7}$ for particles with a diameter of $91 \mathrm{~nm}$ at a particle volume fraction $\phi=7.1 \times 10^{-4}$. Their estimate of $\tilde{Z} \sim 580$ (which is higher than the value of 549 the cell model predicts for the 2040 bare charges on their particles) results from a monodisperse fit to the light scattering data using the rescaled meanspherical closure to calculate the structure factor. Although a monodisperse fit to scattering data from polydisperse samples always underestimates the true effective charge, the overestimation of $\tilde{Z}$ in the work by Versmold et al. results from the underestimation of the colloidal structure by the rescaled mean-spherical approximation.

In the calculation of $\vec{S}(q)$ the charge $Z_{\mu}$ of component $\mu$ was assumed to scale with its surface area, i.e., with $a_{\mu}^{2}$. In order to test the sensitivity of the structure factor with respect to charge polydispersity, we calculated $\bar{S}(q)$ for $Z_{\mu} \propto a_{\mu}$, keeping the average charge constant. The resulting decrease in the charge polydispersity, however, had negligible influence on the width and height of the first peak in $\bar{S}(q)$ (as was also shown in Ref. 21). For the low volume fractions investigated in this study, the height of the first peak in $\bar{S}(q)$ calculated with the Rogers-Young closure differs from the hypernetted-chain result by a few percent, which is within the expcrimental error.

\section{Cell model results}

The experimentally deduced effective charges can now be compared with calculations based on the PoissonBoltzmann cell model (cf. Sec. II C) in the low salt limit, with volume fractions in the range covered by the static light scattering experiments and an effective particle radius $a$ $=\overline{R^{3}} / \overline{R^{2}}=51.3 \mathrm{~nm}$ determined from the corrected size distribution.

Calculations were performed for three different models: the infinite charge model, the mixed sulfate-carboxylate model, and the pure carboxylate model. In the infinite charge model charge regulation is absent. The solution of the Poisson-Boltzmann Eq. (24) yields an effective charge whose saturation value $\tilde{Z}_{\infty}$ depends on volume fraction only. Figure 10 shows that $\tilde{Z}_{\infty}$ first decreases with increasing volume fraction and then reaches a minimum at $\psi \sim 4.0 \times 10^{-3}$. For the particles studied here with $a=51.3 \mathrm{~nm}$ and a Bjerrum length $l_{B}=0.72 \mathrm{~nm}$ the minimum is at $\tilde{Z}_{\infty}=619.5$. At higher volume fractions, however, $\tilde{Z}_{\infty}$ increases again. The infinite charge case could be realized if all carboxylic groups on our particles were replaced by strongly acidic sulfate groups. The sulfate latex particles with $\bar{R}=51 \mathrm{~nm}$ studied recently by Palberg et al. ${ }^{41}$ form colloidal crystals. The number of ionizable sites per particle was determined by conductometric titration. A bare charge of $Z=950$ was found at the volume fraction $\phi=3 \times 10^{-3}$. An effective charge $\tilde{Z}=395$ was found from shear modulus titration at the same volume fraction. This value is, however, significantly lower than the satura- 


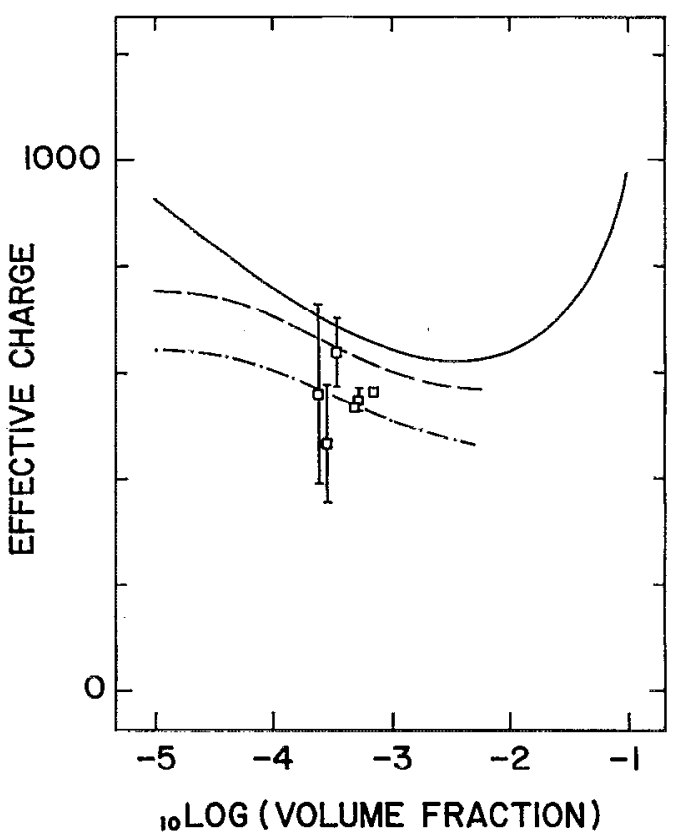

FIG. 10. Measured effective charges for the de-ionized carboxylate polystyrene latex (squares) with the predictions by the Poisson-Boltzmann cell model in the limit of vanishing salt concentration. The effective charges in the mixed model (dashed line) and in the pure model (dash-dotted line) are both below the saturation effective charge predicted by the cell model without charge regulation (full line). The surface chemical parameters used in the cell model calculations were taken from the titration experiments.

tion value $\tilde{Z}_{m}=621$ predicted by the infinite charge cell model, indicating that the saturation is not reached for this number of ionized sites.

In the mixed $(5 \%$ sulfate groups, $95 \%$ carboxylic groups) and the pure carboxylate model we solved numerically the Poisson-Boltzmann equation in the low salt limit including charge regulation (cf. Sec. II C). The surface chemical parameters $\left(p \mathrm{~K}_{\mathrm{COOH}}, C_{S}\right.$, and $\left.\sigma_{t}\right)$ were taken from the titration experiments. We chose low volume fractions in the range where the structure factors had been measured. Figure 10 shows the predictions from the charge regulation models at low salt concentration. Both models predict a decreasing effective charge with $\phi$ increasing from $10^{-5}$ to $10^{-3}$. Both the mixed sulfate-carboxylate and the pure carboxylate model yield effective charges which lie below the saturation value predicted by the infinite charge model. In the mixed sulfate-carboxylate model, the bare surface charge is essentially given by the number of sulfate groups (cf. Table II). It remains constant at about $Z=2000$ as the volume fraction is increased. The number of dissociated carboxylic groups is very small (about $0.045 \%$ ), which is reflected by the low surface $p \mathrm{H}$ of 1.7. Nevertheless the number of sulfate groups is not high enough for the effective charge to reach saturation. ${ }^{42}$

In the pure carboxylate model where no sulfate groups are present, the $p \mathrm{H}$ at the cell boundary differs only slightly from the one in the mixed sulfate-carboxylate model at the same volume fraction. The surface potential, however, is lower in magnitude resulting in a higher $p \mathrm{H}$ at the surface
TABLE II. Predictions of the Poisson-Boltzmann cell for the charge regulation model (carboxylate latex with 5\% sulfate groups) at low salt concentration. The total surface charge density is $\sigma_{t}=-0.2 \mathrm{C} / \mathrm{m}^{2}$, the molar fraction of sulfate groups is $5 \%$, the effective particle radius is $a=51.3 \mathrm{~nm}$. The Stern capacitance is $C_{S}=0.51 \mathrm{~F} / \mathrm{m}^{2}$, and the $p \mathrm{~K}$ of the carboxylic groups is 4.70. The quantity $\alpha=\left[-\mathrm{COO}^{-}\right] /[\mathrm{COOH}]$ is the degree of dissociation of the carboxylic groups.

\begin{tabular}{lcccccc}
\hline \hline$\phi$ & $p \mathrm{H}_{D}$ & $p \mathrm{H}_{0}$ & $Z$ & $\tilde{Z}$ & $\Phi_{0} / \mathrm{mV}$ & $\alpha$ \\
\hline $10^{-5}$ & 6.97 & 1.70 & 2084.2 & 755.9 & -330.4 & $4.64 \times 10^{-4}$ \\
$10^{-4}$ & 6.66 & 1.70 & 2083.9 & 707.1 & -312.2 & $4.58 \times 10^{-4}$ \\
$10^{-3}$ & 5.83 & 1.68 & 2083.3 & 602.5 & -264.6 & $4.43 \times 10^{-4}$ \\
\hline \hline
\end{tabular}

(cf. Table III). Consequently the fraction of dissociated carboxylic groups of about $3 \%$ is by two orders of magnitude larger than in the mixed model.

The effective charges calculated from the charge regulation models show a weaker dependence on volume fraction than in the absence of charge regulation, even in the mixed model where the strongly acidic sulfate groups determine the particle charge. The experimentally determined effective charges (cf. Fig. 10) lie between the limiting value $\tilde{Z}_{\infty}$ in the absence of charge regulation and the prediction from the pure carboxylate model.

However, the relatively small range of volume fractions covered in our light scattering experiments makes it difficult to decide finally which of the two models describes the chemical composition of the surface best. To settle this question one would have to extend the available range of volume fractions using small-angle light scattering techniques. In the range of higher volume fractions, however, the use of scattering techniques is limited since the highly charged particles form crystals. ${ }^{43}$

The generalized cell model including chemical charge regulation, on the other hand, can now be used to predict the effective charge at intermediate salt concentrations or the charging of the particles by addition of strong base such as $\mathrm{NaOH}$. Table IV shows the behavior of the effective charge as the suspension is titrated with $\mathrm{NaOH}$ at constant volume fraction $\phi=10^{-3}$, using the mixed sulfate-carboxylate model. The bare charges increase with addition of $\mathrm{NaOH}$. The corresponding effective charge $\tilde{Z}=671.0$ at the highest $\mathrm{NaOH}$ concentration investigated $\left(4 \times 10^{-5} \mathrm{M}\right)$ is however larger than the saturation value $\tilde{Z}_{\infty}=643.9$ predicted by the infinite charge model. This increase may in fact explain the data by Versmold et al. ${ }^{7}$

TABLE III. Predictions of the Poisson-Boltzmann cell model for the charge regulation model (pure carboxylic latex) at low salt concentration. The surface is composed of carboxylic groups only, with a total surface charge density $\sigma_{t}$ is $-0.11 \mathrm{C} / \mathrm{m}^{2}$ and the average particle radius is $a=51.3 \mathrm{~nm}$. The Stern capacitance is $C_{S}=0.58 \mathrm{~F} / \mathrm{m}^{2}$ and the $p \mathrm{~K}$ of the carboxylic groups is 4.30.

\begin{tabular}{lcccccc}
\hline \hline$\phi$ & $p \mathrm{H}_{D}$ & $p \mathrm{H}_{0}$ & $Z$ & $\tilde{z}$ & $\Phi_{0} / \mathrm{mV}$ & $\alpha$ \\
\hline $10^{-5}$ & 6.98 & 3.00 & 834.6 & 644.0 & -241.4 & $3.67 \times 10^{-2}$ \\
$10^{-4}$ & 6.70 & 2.99 & 816.9 & 606.6 & -225.5 & $3.59 \times 10^{-2}$ \\
$10^{-3}$ & 5.90 & 2.95 & 763.1 & 509.5 & -180.1 & $3.36 \times 10^{-2}$ \\
\hline
\end{tabular}


TABLE IV. Variation of the total and the effective charge in the mixed model ( $5 \%$ sulfate groups present) with added NaOHI at constant volume fraction $\phi=10^{-3}$. The surface chemical parameters are as in Table II.

\begin{tabular}{lcccccc}
\hline \hline$[\mathrm{NaOH}]_{z} / \mathrm{M}$ & $p \mathrm{H}_{D}$ & $p \mathrm{H}_{0}$ & $z$ & $\tilde{Z}$ & $\Phi_{0} / \mathrm{mV}$ & $\alpha$ \\
\hline $10^{-7}$ & 5.84 & 1.69 & 2083.5 & 602.5 & -264.6 & $4.50 \times 10^{-4}$ \\
$10^{-6}$ & 5.91 & 1.76 & 2086.5 & 602.6 & -264.7 & $5.26 \times 10^{-4}$ \\
$10^{-5}$ & 8.02 & 3.64 & 3109.8 & 608.7 & -287.7 & $2.66 \times 10^{-2}$ \\
$4.0 \times 10^{-5}$ & 9.26 & 5.01 & 7094.4 & 671.0 & -317.3 & $1.30 \times 10^{-1}$ \\
\hline \hline
\end{tabular}

\section{CONCLUSIONS}

With the example of highly charged latex particles carrying carboxylic and sulfate groups we have given the experimental evidence that the effective charges describing the interaction of charged colloidal particles at low salt and intermediate salt concentrations are predicted by the charge renormalization procedure. 'This is performed within a generalization of the Poisson-Boltzmann cell model in which the bare charges are computed from the chemical dissociation equilibrium at the particle surface: Effective particle charges were extracted from the analysis of particle structure factors with the polydisperse hypernetted-chain integral equation using a model-free size distribution. This distribution in turn was independently measured with transmission electron microscopy.

Bare particle charges were measured with acid-base titrations at high salt concentrations where interaction between particles can be neglected. The resulting charging curves were best described by a dissociation constant which is very close to the one for the monoprotic acetic acid and a Stern capacitance which may be interpreted as a correction of the mass action law for surface group interactions.

The bare and effective charges were calculated by solving numerically the Poisson-Boltzmann equation in the cell model including the dissociation equilibrium of the carboxylic surface groups and the autodissociation of water. With the inclusion of the charge regulation the resulting effective charges are lower than the saturation values of the effective charges in the absence of charge regulation. The experimentally measured effective charges are in good agreement with the predictions for a mixed sulfate-carboxylate surface. We find that in the low salt regime the effective charges predicted by the cell model are rather insensitive to the surface chemical details (number of ionizable surface groups, dissociation constant and Stern capacitance). This indicates that the concept of charge renormalization retains its general applicability even when chemical charge regulation seems to complicate matters.

\section{ACKNOWLEDGMENTS}

The authors would like to thank S. Egelhaaf and E. Wehrli for the preparation of the TEM pictures, M. Colussi for the thermogravimetric analysis, and A. Lustig for the density gradient measurements. The authors are indebted to
T. Palberg and A. Ferrante for helpful discussions. Financial support from the Swiss National Science Foundation is gratefully acknowledged.

${ }^{1} \mathrm{P}$. N. Pusey, in Liquids, Freezing and Glass Transition, edited by J. P. Hansen, D. Levesque, and J. Zinn-Justin (North-Holland, Amsterdam, 1980).

${ }^{2}$ S. L. Carnie and G. M. Torrie, in Advances in Chemical Physics, edited by I. Prigogine and S. R. Rice (Wiley, New York, 1987), Vol. LVI.

${ }^{3} \mathrm{~J}$. Westall and H. Hohl, Adv. Colloid Interface Sci. 12, 265 (1980).

${ }^{4} \mathrm{~S}$. H. Chen and E. Y. Sheu, in Micellar Solutions and Microemulsions: Structure, Dynamics, and Statistical Thermodynamics, edited by S. H. Chen and R. Rajagopalan (Springer, New York, 1990).

${ }^{5}$ F. Oosawa, Polyelectrolytes (Marcel Dekker, New York, 1971).

${ }^{5}$ S. Bucci, C. Fagotti, V. Degiorgio, and R. Piazza. Langmuir 7, 824 (1991).

${ }^{7}$ H. Versmold, U. Wittig, and W. Härtl, J. Phys. Chem. 95, 9937 (1991); W. Härtl, H. Versmold, and U. Wittig, Langmuir 8, 2885 (1992).

${ }^{8}$ S. Alexander. P. M. Chaikin, P. Grant, G. J. Morales, P. Pincus, and D. Hone, J. Chem. Phys. 80, 5776 (1984).

${ }^{9}$ P. N. Pusey, H. N. W. Lekkerkerker, E. G. D. Cohen, and I. M. De Schepper, Physica A 164, 12 (1990).

${ }^{10} \mathrm{~J}$. P. Hansen and I. R. McDonald, Theory of Simple Liquids (Academic, New York, 1992).

${ }^{11}$ L. S. Ornstein and F. Zernike, Proc. Acad. Sci. (Amsterdam) 17, 793 (1914).

${ }^{12}$ L. Blum, Mol. Phys. 30, 1529 (1975).

${ }^{13}$ L. Blum and J. S. Hóye, J. Phys. Chem. 81, 1311 (1977).

${ }^{14}$ F. R. Rogers and D. A. Young, Phys. Rev. A 30, 999 (1984).

${ }_{15}$ M. Medina-Noyola and D. A. McQuarrie, J. Chem. Phys. 73, 6279 (1980).

${ }^{16}$ E. J. W. Verwey and J. Th. G. Overbeek, Theory of the Stability of Lyophobic Colloids (Elsevier, New York, 1948).

${ }^{17}$ L. Belloni, J. Chem. Phys. 85, 519 (1986).

${ }^{18}$ L. Belloni, J. Chem. Phys, 98, 8080 (1993).

${ }^{19}$ H. Löwen, J. P. Hansen, and P. A. Madden, J. Chem. Phys. 98, 3275 (1993).

${ }^{20} \mathrm{H}$. Löwen and G. Kramposthuber, Europhys. Lett. 23, 673 (1993).

${ }^{21}$ B. D'Aguanno and R. Klein, J. Chem. Soc. Faraday Trans. 87, 379 (1991).

${ }^{22}$ W. B. Russel, D. A. Saville, and W. R. Schowalter, Colloidal Dispersions (Cambridge University, Cambridge, 1989).

${ }^{23}$ J. C. M. de Wit, W. H. van Riemsdijk, M. M. Nederlof, D. G. Kinniburgh, and L. K. Koopal, Analytica Chimica Acta 232, 189 (1990).

${ }^{24}$ S. F. Schulz, T. Gisler, M. Borkovec, and H. Sticher, J. Colloid Interface Sci. 164, 88 (1994).

${ }^{25}$ L. Belloni, Chem. Phys. 99, 43 (1985).

${ }^{26} \mathrm{M}$. Fushiki, J. Chem. Phys. 97, 6700 (1992).

${ }^{27}$ J. P. H. Zwetsloot and J. C. Leyte, J. Colloid Interface Sci. 163, 362 (1994).

${ }^{28} \mathrm{P}$. Schurtenberger and M. E. Newman, in Environmental Particles, edited by J. Buffle, H. P. van Leeuwen (Lewis, Boca Raton, 1993).

${ }^{29}$ N. J. Wagner, R. Krause, A. R. Rennie, B. D'Aguanno, and J. Goodwin, J. Chem. Phys. 95, 494 (1991).

${ }^{30}$ S. J. Gregg and K. S. W. Singh, Adsorption, Surface Area and Porosity (Academic, New York, 1982).

${ }^{31}$ A. Delgado and E. Matijevic, Part. Part. Syst. Charact. 8, 128 (1991).

${ }^{32}$ The specific surface area $S$ area was calculated from the size distribution by $S=3 R^{2} /\left(\rho_{\text {Latex }} R^{3}\right)$; using the density of the polystyrene latex particles $\rho_{\text {Latex }}=1.043 \mathrm{~g} / \mathrm{cm}^{3}$.

${ }^{33}$ T. H. Hill, J. Am. Chem. Soc. 78, 5527 (1956).

${ }^{34}$ J. K. Dhont, Physica 120A, 238 (1983).

${ }^{35}$ The NAG Fortran Library Manual, Mark 15 (1991).

${ }^{36}$ D. Shanks, Studies Appl. Math. 34, 1 (1955).

${ }^{37}$ T. Palberg, J. Kottal, F. Bitzer, R. Simon, M. Würth, and P. Leiderer, J. Colloid Interface Scì. (submitted).

${ }^{38}$ For the Stern capacitance $C_{S}=0.51 \mathrm{~F} \mathrm{~m}^{-2}$ used in the cell model calculation for the mixed sulfate-carboxylate case, the potential difference across the Stern layer is of the order of $20 \mathrm{mV}$ (which is about $10 \%$ of the value of $\Psi_{0}$ ). Thus the structure of the electric double layer at low salt concentrations appears to be well described by the simple diffuse layer model.

${ }^{39}$ Y. Monovoukas and A. P. Gast, J. Colloid Interface Sci. 128, 533 (1989), 